\title{
Prescribing health information - the development and evaluation of a smartphone app providing guidance and management advice for a feverish child
}

\author{
Authors: Aisling Chew, Aaron Koshy and Emma Lim
}

\begin{abstract}
Aims
To develop a smartphone app-based educational module for parents and carers looking after children with fever. Following deployment, to evaluate the use and acceptability of the appbased fever module compared with a traditional leaflet.
\end{abstract}

\section{Methods}

An educational multi-platform app (Pocket Health) was created with reliable up-to-date medical information in an accessible format. The app was developed by clinicians with a specific module written for the care of febrile children in response to requests for more mobile-based health information. The module underwent multiple rounds of parent-led qualitative feedback.

Between December 2017 and January 2018, the feverish child module and corresponding patient information leaflet were provided to parents and carers of children with fever presenting to the Great North Children's Hospital, Newcastle-upon-Tyne's emergency department (ED). Feedback was also received directly from responders after distributing the app via local parent patient advocates. Quantitative and qualitative feedback was obtained through an online questionnaire created for this study.

\section{Results}

App access and leaflets were distributed to 48 members of the public. A total of 47 responses ( 25 and 22 for the module and leaflet respectively) were obtained through the online questionnaire.

The module was rated a mean score of 4.76 out of 5 for helpfulness, compared with 4.22 for the leaflet. $100 \%$ of participants in the app and $90.9 \%$ of those receiving the leaflet felt more confident after reading the information. $60.0 \%$ vs $54.5 \%$ in the app and leaflet groups respectively felt they were less likely to make an urgent appointment with their general practitioner or attend ED after receiving the information.

\section{Conclusion}

Fever is among the commonest reasons that children present acutely to healthcare services. There is little high-quality, credible, evaluated app-based information on acute childhood illnesses. We developed this app at the request of families and tailored it around their feedback. The content was rated highly by parents and carers and has been shown to improve confidence. Additional benefits are the perception of reduced need for access to healthcare.

We believe that the parent-led demand for credible high-quality, reliable and tailored advice will drive an increase in smartphonebased health apps. However, this must be underpinned by clear evidence-based guidelines and research to provide best quality information.

\section{Conflict of interest statement}

Dr Koshy is involved in the development of Pocket Health. All study members moderated the quality of content given to the participants. 